\title{
Estratégias de prevenção ou redução do consumo de drogas para adolescentes:
} revisão sistemática da literatura

Thamyris Alexandre Salles ${ }^{1}$, Erika de Cássia Lopes Chaves ${ }^{2}$, Denis da Silva Moreira ${ }^{3}$, Mariana Viotti Nogueira Brito $^{4}$, Haze Maria Carolina Risolia e Mendonça ${ }^{5}$, Keila Oliveira ${ }^{6}$

\footnotetext{
${ }^{1}$ Enfermeira, Mestre em Enfermagem. Alfenas, MG, Brasil. E-mail: thamyrissalles19@hotmail.com.

${ }^{2}$ Enfermeira, Doutora em Enfermagem Fundamental. Professora Adjunto da Universidade Federal de Alfenas. Alfenas, MG Brasil. E-mail: erika.chaves@unifal-mg.edu.br.

${ }^{3}$ Enfermeiro, Doutor em Enfermagem. Professor Adjunto da Universidade Federal de Alfenas. Alfenas, MG, Brasil. E-mail: denisunifal@gmail.com.

${ }^{4}$ Discente do curso de graduação em Enfermagem da Universidade Federal de Alfenas. Alfenas, MG, Brasil. E-mail: mariviotti.mv@gmail.com.

${ }^{5}$ Enfermeira. Alfenas, MG, Brasil. E-mail: haze_risolia@hotmail.com.

${ }^{6}$ Discente do curso de graduação em Enfermagem da Universidade Federal de Alfenas. Alfenas, MG, Brasil. E-mail: keila2717@gmail.com.
}

Recebido: 21/07/2015.

Aceito: 14/03/2016.

Publicado: 30/06/2016.

Como citar esse artigo:

Salles TA, Chaves ECL, Moreira DS, Brito MVN, Mendonça HMCR, Oliveira K. Estratégias de prevenção ou redução do consumo de drogas para adolescentes: revisão sistemática da literatura. Rev. Eletr. Enf. [Internet]. 2016 [acesso em:____ 18:e1172. Disponível em: http://dx.doi.org/10.5216/ree.v18.36796

\section{RESUMO}

Foi realizada uma revisão sistemática com o objetivo de identificar as evidências científicas acerca das estratégias de prevenção ou redução do consumo de drogas entre adolescentes. A busca foi realizada nas bases de dados LILACS, CINAHL, MEDLINE, Scopus e Cochrane Library, com os descritores Ensino, Educação em Saúde, Transtornos relacionados ao uso de substâncias, Adolescentes e Enfermagem Psiquiátrica. Foram selecionados 27 artigos. Os resultados demonstraram uma variedade de programas, projetos e intervenções utilizados como estratégias de prevenção ou redução do consumo de drogas entre adolescentes. Dentre os estudos, $74,1 \%(n=20)$ observaram resultado satisfatório e $25,9 \% \quad(n=7)$ parcialmente satisfatório. Apenas o Projeto Narconon e a Intervenção Breve demonstraram efetividade, de maneira ampla, na redução do consumo de diferentes tipos de drogas entre adolescentes, uma vez que as demais foram direcionadas à algum tipo específico de drogas ou ainda se limitaram à prevenção do consumo destas sustâncias à adolescentes não usuários.

Descritores: Ensino; Educação em Saúde; Transtornos Relacionados ao Uso de Substâncias; Adolescentes; Enfermagem Psiquiátrica.

\section{INTRODUÇÃO}

Nos dias atuais, o uso de drogas é considerado pela Organização Mundial de Saúde (OMS) um grave 
problema de saúde pública, uma vez que ocasiona numerosas repercussões sociais, físicas e mentais(1). Ainda, segundo a OMS, aproximadamente $10 \%$ dos moradores urbanos do mundo inteiro, com diferentes idades, sexo, escolaridade ou renda, fazem uso exagerado de substâncias psicoativas(2).

No cenário internacional uma pesquisa realizada no México mostrou que mais de 200.000 adolescentes na faixa etária dos 12 aos 17 anos de idade estão envolvidos com a utilização de drogas, sendo que idade de início do consumo em média, foi aos 14 anos. Em relação ao gênero observa-se que para cada usuário do sexo feminino há 3,5 usuários do sexo masculino. Estes achados demonstram que o uso de drogas constitui uma prática mais frequente na população de adolescentes(3).

O Centro Brasileiro de Informações sobre Drogas Psicotrópicas-CEBRID, também evidenciou no seu último levantamento, publicado em 2010, em que participaram 50.890 estudantes do ensino fundamental e médio, de 27 capitais brasileiras, que 25,5\% dos adolescentes referiam uso, na vida, de alguma droga(4).

Um estudo também retratou que a utilização de drogas está cada vez mais precoce em razão da vulnerabilidade dos adolescentes, o que ocasiona maior probabilidade de gerar problemas, tais como: dependências; acidentes de trânsito; violência; conflitos familiares; problemas de trabalho; doenças; entre outros(5). O consumo de substâncias que alteram o estado mental está contido no percurso histórico da humanidade; pois está relacionado a questões culturais ou religiosas, a momentos recreativos, a mecanismo de lidar com problemas e como recurso de socialização ou de isolamento(6).

O uso abusivo de drogas também gera custos sociais elevados, o que demanda a execução de ações corretas em saúde pública(7). Desse modo, o consumo de drogas constitui um relevante problema que requer a atenção de todos, ou seja, da sociedade em geral, dos profissionais de saúde, da comunidade científica e dos educadores.

Nos últimos tempos, as ações voltadas para o uso de substâncias psicoativas têm progredido de um padrão em que eram focadas no tratamento e na intervenção, para valorizar a educação, a saúde, a vida e a contribuição familiar, ou seja, passam a ser direcionadas a estratégias de prevenção(8). Como demonstrado em alguns estudos o ambiente escolar, onde geralmente o adolescente se encontra inserido, constitui um espaço favorável para realização dessas estratégias, pois sua função fundamental de educar para a vida, propicia melhor entendimento das coisas e pode gerar mudanças sociais para um futuro melhor para esses jovens(9). Para intervir como elemento de proteção contra o uso de drogas o campo do ensino deve se articular com o da saúde, para que juntos estabeleçam ações a ser desenvolvidas(3).

Nessa perspectiva, são relevantes estudos que evidenciam ações de prevenção ou redução eficazes para uma tomada de decisão, e que direcionem a sociedade em geral na escolha da melhor estratégia de prevenção ou redução do consumo de álcool e de outras drogas, a ser empregada para a população de adolescentes, a qual seja capaz de colaborar para minimização da problemática de utilização precoce de drogas e de suas consequências.

Assim, este estudo teve por objetivo identificar as estratégias de prevenção ou redução do consumo de drogas direcionadas à população de adolescentes. 


\section{MÉTODO}

Revisão sistemática da literatura, a qual consiste em uma síntese rigorosa de todos os estudos relacionados a um determinado tema, envolvendo geralmente a efetividade de uma intervenção. Ela também propicia o agrupamento e a análise dos resultados decorrentes de pesquisas primárias ${ }^{(10)}$. A presente revisão foi desenvolvida de acordo com os critérios do PRISMA Statement (Preferred Reporting Items for Systematic Reviews and Meta-Analyses), o qual contém 27 itens de uma lista de verificação que englobam informações que devem ser incluídas em revisões sistemáticas e o fluxograma de seleção dos $\operatorname{artigos}^{(11)}$.

Para atingir o objetivo foram definidas as seguintes questões: Quais estratégias são utilizadas para a prevenção do consumo de álcool e/ou de outras drogas na população de adolescentes? Quais os objetivos das estratégias? Onde, por quem e como são desenvolvidas? Qual sua efetividade?

Foi realizada a busca nas bases de dados Literatura Latino-Americana e do Caribe em Ciências da Saúde (LILACS), Cumulative Index to Nursing and Allied Health Literature (CINAHL), Pubmed/MEDLINE, Scopus e na Cochrane Library. utilizando os seguintes descritores, separados pelo operador booleano and:"Ensino/Teaching", "Educação em Saúde/Health education", "Transtornos relacionados ao uso de substâncias/Substance related disorders" "Adolescentes/Adolescent" e "Enfermagem Psiquiátrica /Psychiatric Nursing".

Os critérios de elegibilidade para a seleção dos artigos foram: estudos experimentais e quase experimentais que aplicaram alguma estratégia de prevenção do uso de álcool e outras drogas entre adolescentes e que obtiveram evidencias científicas acima de III, conforme classificação Stetler et al ${ }^{(12)}$, publicados a partir de 2003, pois foi quando foi instituída a política antidrogas ${ }^{(13)}$, nos idiomas português, inglês e espanhol e com resumo disponível nas bases de dados para a apreciação.

A coleta de dados ocorreu nos meses de fevereiro a março de 2015 , seguida de uma avaliação minuciosa dos estudos encontrados, que abrangeu uma leitura exploratória, seletiva, analítica e interpretativa $^{(11)}$.

Inicialmente foram identificadas 991 referências potenciais para esta revisão; destas, 74 artigos foram selecionados para a leitura crítica na íntegra e após, foram excluídos 47 artigos em razão de duplicidade ou por não responderem as questões norteadoras do estudo; ao final foram eleitos 27 artigos para a análise. A Figura 1 demonstra a síntese do processo de seleção dos artigos. 

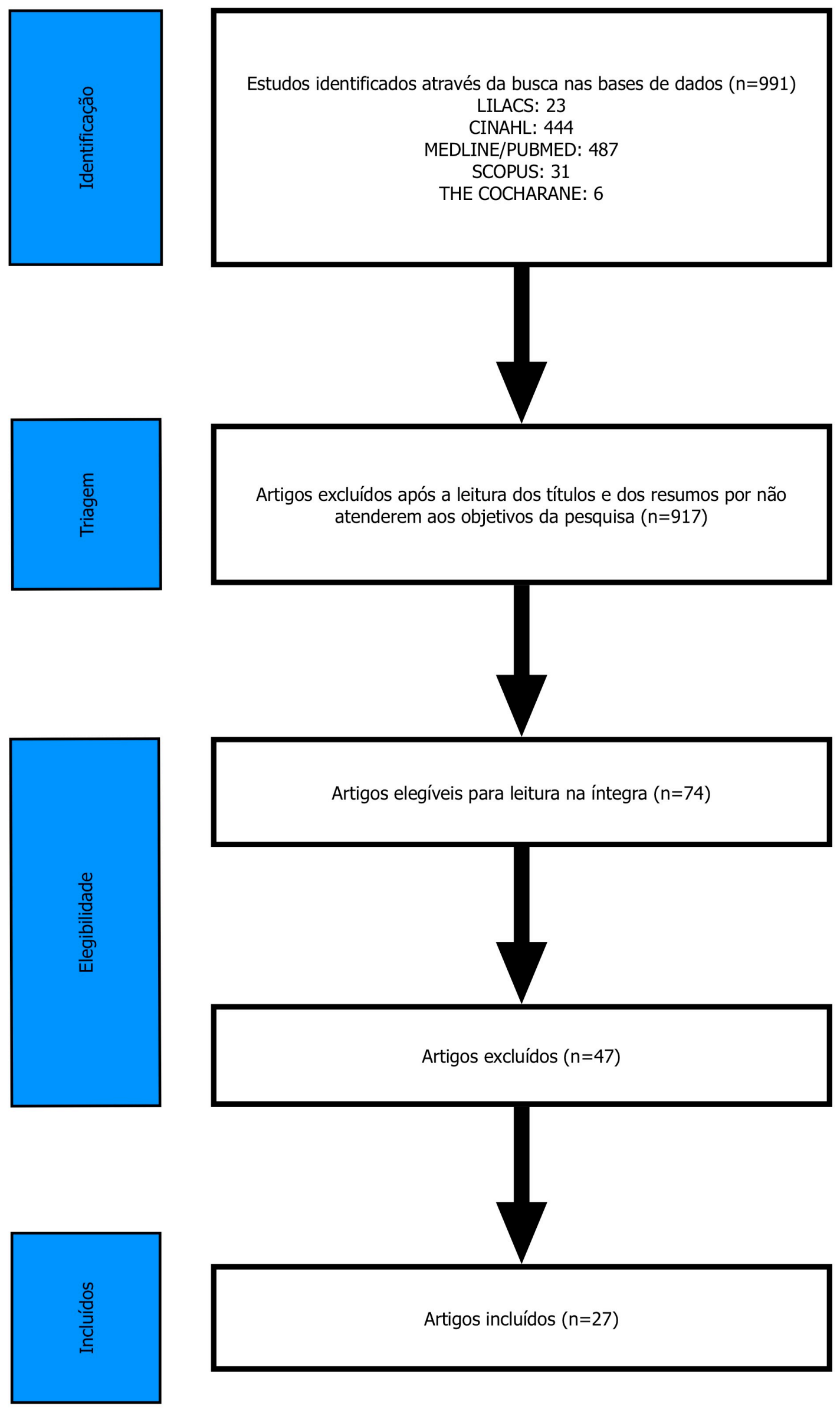

Figura 1: Fluxograma de seleção dos artigos. 
Para a avaliação dos estudos os dados foram organizados em um instrumento ${ }^{(10)}$, composto pelos seguintes itens: Autores/Ano de Publicação; País de Origem; Método-nível de evidência, Estratégia Utilizada; Objetivo da Estratégia; Local onde foi realizada a estratégia e quem realizou e Efetividade. A análise crítica dos resultados que os artigos apresentavam ocorreu em dois momentos. No primeiro momento, para apresentar as características dos artigos encontrados e, no segundo, para realizar a análise do conteúdo dos artigos.

\section{RESULTADOS}

O Quadro 1 apresenta as características dos estudos incluídos na revisão sistemática quanto às estratégias de prevenção ou redução sobre álcool e outras drogas direcionadas a população de adolescentes. Em relação ao tipo de estudo $100 \%$ deles foram experimentais e com nível de evidência ${ }^{(12)}$. Quanto ao ano em que os artigos foram publicados a maioria foi desenvolvida em 2013 e o país de publicação que se destaca com um maior número de artigos são os Estados Unidos (44,4\%). 
Quadro 1: Características dos estudos incluídos na revisão sistemática e quanto a síntese das estratégias de prevenção sobre álcool e outras drogas direcionadas a população de adolescentes.

\begin{tabular}{|c|c|c|c|c|c|c|}
\hline $\begin{array}{l}\text { Autores, ano de } \\
\text { Publicação }\end{array}$ & Pais de origem & $\begin{array}{l}\text { Método-nível } \\
\text { de evidencia* }\end{array}$ & Estratégia Utilizada & Objetivo da estratégia & $\begin{array}{c}\text { Local/quem realizou a } \\
\text { estratégia }\end{array}$ & Efetividade da Estratégia \\
\hline $\begin{array}{l}\text { Giannotta et al, 2013 }{ }^{(14)} \\
\text { Faggiano et al, 2010 } \\
\text { Caria et al, } 2011^{(16)} \\
\text { Gabrhelik et al, } 2012^{(17)}\end{array}$ & $\begin{array}{l}{ }^{(14-16)} \text { Sete Países } \\
\text { Europeus } \\
\text { (Áustria, Bélgica, } \\
\text { Alemanha, Grécia, } \\
\text { Itália, Espanha, } \\
\text { Suécia). } \\
{ }^{(17)} \text { República } \\
\text { Tcheca }\end{array}$ & $\begin{array}{l}{ }^{(14-17)} \text { Estudos } \\
\text { Experimentais- } \\
\text { nível II }\end{array}$ & $\begin{array}{c}\text { Programa com Curriculum } \\
\text { Unplugged }\end{array}$ & $\begin{array}{l}\text { Prevenir ou reduzir o uso } \\
\text { de drogas. }\end{array}$ & Escolas/professores & $\begin{array}{l}\text { Demonstrou efetividade } \\
\text { parcial em razão de } \\
\text { impactar na redução do } \\
\text { consumo de apenas uma } \\
\text { determinada droga. }\end{array}$ \\
\hline $\begin{array}{l}\text { Koning et al, } 2013^{(18)} \\
\text { Koning et al, } 2012^{(19)}\end{array}$ & ${ }^{(18-19)}$ Holanda & $\begin{array}{l}{ }^{(18-19)} \text { Estudos } \\
\text { Experimentais- } \\
\text { nível II }\end{array}$ & $\begin{array}{l}\text { Programa de Prevenção } \\
\text { Universal do Uso de álcool para } \\
\text { adolescentes e seus pais (PAS) }\end{array}$ & $\begin{array}{l}\text { Reduzir o uso pesado e } \\
\text { precoce de álcool nos finais } \\
\text { de semana. }\end{array}$ & $\begin{array}{c}\text { Escolas/ assistentes } \\
\text { treinados }\end{array}$ & $\begin{array}{l}\text { Foi eficaz em reduzir o } \\
\text { consumo de álcool. }\end{array}$ \\
\hline $\begin{array}{l}\text { Sussman et al,2003 } \\
\text { Rohrbach;Sussman,2010 } \\
\text { (21) }\end{array}$ & $\begin{array}{c}{ }^{(20-21)} \text { Estados } \\
\text { Unidos }\end{array}$ & $\begin{array}{c}\text { (20-21) } \\
\text { Estudos } \\
\text { Experimentais- } \\
\text { nível II }\end{array}$ & $\begin{array}{l}\text { Projeto Rumo sem abuso de } \\
\text { drogas-TND }\end{array}$ & Prevenir o abuso de drogas & $\begin{array}{c}\text { Escolas/ professores } \\
\text { treinados }\end{array}$ & $\begin{array}{l}\text { Demonstrou efetividade } \\
\text { parcial em razão de } \\
\text { impactar na redução do } \\
\text { consumo de apenas uma } \\
\text { determinada droga. }\end{array}$ \\
\hline Lennox; Cecchini, $2008^{(22)}$ & Estados Unidos & $\begin{array}{c}\text { Estudo } \\
\text { Experimental- } \\
\text { nível II }\end{array}$ & Programa Narconon & $\begin{array}{l}\text { Prevenir o consumo de } \\
\text { drogas nas escolas. }\end{array}$ & $\begin{array}{l}\text { Escolas/ facilitadores } \\
\text { treinados profissionalmente }\end{array}$ & $\begin{array}{l}\text { Demonstrou efetividade na } \\
\text { redução do uso de drogas. }\end{array}$ \\
\hline Huang xet al, 2011 & Taiwan & $\begin{array}{l}\text { Estudo } \\
\text { Experimental- } \\
\text { nível II }\end{array}$ & $\begin{array}{l}\text { Programa de Prevenção do Uso } \\
\text { de Drogas integrando a Teoria } \\
\text { do Comportamento Planejado } \\
\text { e Habilidades de Vida }\end{array}$ & Prevenir o uso de drogas & Escolas /professores & $\begin{array}{l}\text { Foi eficaz, pois melhorou as } \\
\text { atitudes, comportamentos e } \\
\text { habilidades de vida dos } \\
\text { adolescentes na intenção de } \\
\text { não usar drogas }\end{array}$ \\
\hline Sloboda et al,2009 (24) & Estados Unidos & $\begin{array}{l}\text { Estudo } \\
\text { experimental- } \\
\text { nível II }\end{array}$ & $\begin{array}{l}\text { Programa de Prevenção } \\
\text { Universal em meio escolar se } \\
\text { encarregar de sua vida (TCYL) }\end{array}$ & $\begin{array}{l}\text { Impedir ou reduzir o uso de } \\
\text { álcool tabaco e maconha. }\end{array}$ & Escolas/Professores & $\begin{array}{l}\text { Demonstrou efetividade } \\
\text { parcial em razão de } \\
\text { impactar na redução do } \\
\text { consumo de apenas uma } \\
\text { determinada droga. }\end{array}$ \\
\hline
\end{tabular}




\begin{tabular}{|c|c|c|c|c|c|c|}
\hline $\begin{array}{l}\text { Autores, ano de } \\
\text { Publicação }\end{array}$ & Pais de origem & $\begin{array}{l}\text { Método-nível } \\
\text { de evidencia* }\end{array}$ & Estratégia Utilizada & Objetivo da estratégia & $\begin{array}{c}\text { Local/quem realizou a } \\
\text { estratégia }\end{array}$ & Efetividade da Estratégia \\
\hline Perry et al,2007 ${ }^{(25)}$ & Estados Unidos & $\begin{array}{l}\text { Estudo } \\
\text { experimental- } \\
\text { nível II }\end{array}$ & Projeto Northland & $\begin{array}{l}\text { Prevenir ou retardar o uso } \\
\text { de álcool entre } \\
\text { adolescentes. }\end{array}$ & Escolas/professores & $\begin{array}{l}\text { O projeto impactou na } \\
\text { redução do uso de álcool } \\
\text { entre adolescentes e no } \\
\text { crescimento de problemas } \\
\text { familiares. }\end{array}$ \\
\hline $\begin{array}{l}\text { Eisen; Zellman; } \\
\text { Murray, } 2003^{(26)}\end{array}$ & Estados Unidos & $\begin{array}{l}\text { Estudo } \\
\text { experimental- } \\
\text { nível II }\end{array}$ & $\begin{array}{l}\text { Habilidades para adolescência- } \\
\text { SFA }\end{array}$ & $\begin{array}{c}\text { Prevenir ou retardar o uso } \\
\text { de tabaco, álcool e drogas } \\
\text { ilegais }\end{array}$ & Escolas/professores & $\begin{array}{l}\text { As habilidades de vida na } \\
\text { adolescência foram eficazes } \\
\text { em prevenir o uso de } \\
\text { substâncias entre } \\
\text { adolescentes. }\end{array}$ \\
\hline $\begin{array}{l}\text { Micheli; Fisberg; } \\
\text { Formigoni,2004 } \\
\text { Goti et al,2010 } \\
\text { Patten et al,2006 } \\
\text { Doumas et al, } 2014^{(29)}\end{array}$ & $\begin{array}{l}{ }^{(27)} \text { Brasil } \\
{ }^{(28)} \text { Espanha } \\
{ }^{(29-30)} \text { Estados } \\
\text { Unidos }\end{array}$ & $\begin{array}{l}\text { Estudos } \\
\text { Experimentais- } \\
\text { nível II }\end{array}$ & Intervenção Breve & $\begin{array}{l}\text { Prevenir ou reduzir o uso } \\
\text { de álcool e outras } \\
\text { drogas. } \\
\text { Aumentar o conhecimento } \\
\text { sobre os riscos do uso de } \\
\text { substâncias. }{ }^{(26)} \\
\text { Cessar o tabagismo entre } \\
\text { adolescentes. }{ }^{(27)}\end{array}$ & $\begin{array}{c}\text { * Centro de Apoio e } \\
\text { atendimento ao } \\
\text { adolescente/ quatro } \\
\text { médicas pediatras. } \\
\text { *Serviço psiquiátrico/ } \\
\text { profissionais deste serviço. } \\
\text { * Na web/ educador de } \\
\text { saúde. }\end{array}$ & $\begin{array}{c}\text { Promoveu reforço } \\
\text { motivacional nos } \\
\text { adolescentes e colaborou } \\
\text { para redução do uso de } \\
\text { drogas. }\end{array}$ \\
\hline Baer et al,2008 ${ }^{(31)}$ & Estados Unidos & $\begin{array}{l}\text { Estudo } \\
\text { Experimental- } \\
\text { nível II }\end{array}$ & Motivacional & $\begin{array}{l}\text { Gerar mudanças de } \\
\text { comportamento no } \\
\text { adolescente como o uso de } \\
\text { substâncias. }\end{array}$ & Centro social/ profissionais & $\begin{array}{l}\text { Foi eficaz ao provocar } \\
\text { mudanças de } \\
\text { comportamento do } \\
\text { adolescente em relação ao } \\
\text { uso de substâncias. }\end{array}$ \\
\hline Gregor et al, $2003^{(32)}$ & Estados Unidos & $\begin{array}{l}\text { Estudo } \\
\text { Experimental- } \\
\text { nível II }\end{array}$ & Programa Interativo Portátil & $\begin{array}{l}\text { Evitar o uso indevido de } \\
\text { álcool entre adolescentes. }\end{array}$ & $\begin{array}{c}\text { Serviço de } \\
\text { urgência/profissionais. }\end{array}$ & $\begin{array}{l}\text { O programa foi eficaz } \\
\text { levando o adolescente a } \\
\text { repensar sobre o consumo } \\
\text { de álcool. }\end{array}$ \\
\hline Werch et al,2005 ${ }^{(33)}$ & Estados Unidos & $\begin{array}{l}\text { Estudo } \\
\text { Experimental- } \\
\text { nível II }\end{array}$ & $\begin{array}{l}\text { Intervenção nos múltiplos } \\
\text { comportamentos de saúde } \\
\text { com a integração de atividade } \\
\text { física à prevenção do uso de } \\
\text { substâncias }\end{array}$ & $\begin{array}{l}\text { Prevenir e reduzir o uso de } \\
\text { álcool e outras drogas e } \\
\text { aumentar a realização de } \\
\text { atividades físicas entre } \\
\text { adolescentes. }\end{array}$ & Escola/equipe treinada & $\begin{array}{c}\text { Essa estratégia demonstrou } \\
\text { efetividade na prevenção do } \\
\text { uso de substâncias }\end{array}$ \\
\hline
\end{tabular}




\begin{tabular}{|c|c|c|c|c|c|c|}
\hline $\begin{array}{l}\text { Autores, ano de } \\
\text { Publicação }\end{array}$ & Pais de origem & $\begin{array}{l}\text { Método-nível } \\
\text { de evidencia* }\end{array}$ & Estratégia Utilizada & Objetivo da estratégia & $\begin{array}{c}\text { Local/quem realizou a } \\
\text { estratégia }\end{array}$ & Efetividade da Estratégia \\
\hline Conrod et al, $2013^{(34)}$ & Reino Unido & $\begin{array}{l}\text { Estudo } \\
\text { Experimental- } \\
\text { nível II }\end{array}$ & $\begin{array}{l}\text { Intervenção direcionada à } \\
\text { personalidade da juventude ou } \\
\text { tratamento HR (Educação Legal } \\
\text { sobre drogas) }\end{array}$ & $\begin{array}{l}\text { Prevenir o uso indevido de } \\
\text { álcool. }\end{array}$ & $\begin{array}{c}\text { Escola/Professores } \\
\text { Treinados }\end{array}$ & $\begin{array}{l}\text { A Estratégia conferiu efeitos } \\
\text { positivos na redução do } \\
\text { álcool. }\end{array}$ \\
\hline Serrano et al, $2013^{(35)}$ & Espanha & $\begin{array}{l}\text { Estudo } \\
\text { Experimental- } \\
\text { nível II }\end{array}$ & Programa Saluda & $\begin{array}{l}\text { Prevenir e promover a } \\
\text { saúde dos adolescentes em } \\
\text { relação ao uso de álcool e } \\
\text { outras drogas. }\end{array}$ & $\begin{array}{l}\text { Escola/ estudantes de } \\
\text { graduação de psicologia }\end{array}$ & $\begin{array}{c}\text { O programa é muito } \\
\text { eficiente na prevenção e } \\
\text { promoção de saúde para } \\
\text { adolescentes no que tange } \\
\text { ao uso de álcool e outras } \\
\text { drogas. }\end{array}$ \\
\hline Guo et al,2010 ${ }^{(36)}$ & China & $\begin{array}{l}\text { Estudo } \\
\text { Experimental- } \\
\text { nível II }\end{array}$ & $\begin{array}{l}\text { Programa de intervenção em } \\
\text { Saúde baseada na escola- } \\
\text { Motivação Cognitiva, } \\
\text { Inteligência Emocional e } \\
\text { Habilidades de Resistência na } \\
\text { Prevenção do uso de drogas }\end{array}$ & $\begin{array}{l}\text { Melhorar a cognição sobre } \\
\text { o uso de drogas para } \\
\text { diminuir a motivação do } \\
\text { uso e melhorar as } \\
\text { habilidades de resistência } \\
\text { às drogas. }\end{array}$ & Escola/professores & $\begin{array}{l}\text { O programa foi eficaz na } \\
\text { prevenção do abuso de } \\
\text { drogas. }\end{array}$ \\
\hline Walton et al,2013 & Estados Unidos & $\begin{array}{l}\text { Estudo } \\
\text { Experimental- } \\
\text { nível II }\end{array}$ & $\begin{array}{l}\text { Intervenções Breves Baseadas } \\
\text { no Computador (CBI) e no } \\
\text { terapeuta (TCE) entre usuários } \\
\text { de cannabis }\end{array}$ & $\begin{array}{l}\text { Diminuir os problemas } \\
\text { relacionados ao uso de } \\
\text { cannabis }\end{array}$ & $\begin{array}{l}\text { Serviço de atenção } \\
\text { primária/enfermeira }\end{array}$ & $\begin{array}{c}\text { A Intervenção Breve } \\
\text { desenvolvida no } \\
\text { computador diminui o uso } \\
\text { de cannabis entre } \\
\text { adolescentes e os problemas } \\
\text { relacionados. }\end{array}$ \\
\hline $\begin{array}{l}\text { Toumbourou et al,2013 } \\
\text { Milburn et al,2012 }\end{array}$ & $\begin{array}{l}{ }^{(38)} \text { Austrália } \\
{ }^{(39)} \text { Estados Unidos }\end{array}$ & $\begin{array}{l}{ }^{(38-39)} \text { Estudos } \\
\text { Experimentais- } \\
\text { nível II }\end{array}$ & Intervenção Familiar & $\begin{array}{l}\text { Reduzir o consumo de } \\
\text { álcool na adolescência. }\end{array}$ & $\begin{array}{c}\text { *Escolas/ professores } \\
\text { * Organização de base } \\
\text { comunitária/ um facilitador } \\
\text { treinado }\end{array}$ & $\begin{array}{l}\text { Os adolescentes } \\
\text { demonstraram redução do } \\
\text { consumo dessa substância. }\end{array}$ \\
\hline Primack et al,2014 ${ }^{(40)}$ & Estados Unidos & $\begin{array}{l}\text { Estudo } \\
\text { Experimental- } \\
\text { nível II }\end{array}$ & $\begin{array}{l}\text { Currículo de literacia mediática } \\
\text { anti-tabagismo }\end{array}$ & $\begin{array}{l}\text { Reduzir o consumo do } \\
\text { tabagismo entre } \\
\text { adolescentes. }\end{array}$ & Escolas/professores & $\begin{array}{c}\text { A estratégia melhorou a } \\
\text { percepção dos jovens sobre } \\
\text { o tabagismo }\end{array}$ \\
\hline
\end{tabular}

* Stetler et al., 1998. 
Os estudos analisados exploraram 18 diferentes estratégias de prevenção ou redução do consumo de drogas direcionadas à população de adolescentes, que envolviam desde a utilização de programas, de projetos até intervenções. Dos 27 estudos, ao explorar o local e quem realizou a estratégia de prevenção de álcool e de outras drogas direcionadas à população de adolescentes, foi encontrado que: $74,1 \%(n=20)$ dos estudos foram realizados na escola por professores ou por pessoas treinadas; $3,7 \%(n=1)$ no Centro de Apoio e Atendimento ao Adolescente (CAAA) e por quatro médicas pediatras; $3,7 \%(n=1)$ no centro social por profissionais; $3,7 \%(n=1)$ dos estudos por profissionais de um serviço de urgência; $3,7 \%(n=1)$ por profissionais de um serviço psiquiátrico; $3,7 \%(n=1)$ por uma enfermeira em um serviço de atenção primária; 3,7\% ( $n=1)$ por um educador de saúde e um profissional treinado online e 3,7\%(n=1) por um facilitador treinado em uma organização de base comunitária.

Ao analisar a efetividade das estratégias de prevenção ou redução do consumo de álcool e de outras drogas direcionadas à população de adolescentes, foi encontrado que $74,1 \%(n=20)$ dos estudos demonstraram resultado satisfatório para prevenir ou para reduzir o consumo de álcool e/ou de outras drogas entre adolescentes e $25,9 \%$ ( $n=7$ ) dos estudos demonstraram efetividade parcial, uma vez que obteve efeito satisfatório apenas em algumas das substâncias investigadas.

Dentre as limitações descritas em estudo que apresentou efetividade parcial em relação a estratégia de prevenção do consumo de drogas aplicada, encontram-se a diversidade geográfica da amostra, habilidades de recusa, presença de crenças normativas e apresentação de pequenos efeitos de mediação(14).

\section{DISCUSSÃO}

Este estudou confirma a importância da aplicação de estratégias de prevenção ou redução do consumo de álcool e/ou de outras drogas em adolescentes; uma vez que todas as estratégias investigadas sejam elas programas, projetos ou intervenções favoreceram a prevenção ou redução, de forma integral $(74,1 \%)$ ou parcial $(25,9 \%)$, do consumo destas substâncias.

Em alguns estudos ${ }^{(14-17,20-21,24)}$, as estratégias investigadas demonstraram efetividade parcial devido à falta de resultado na redução do consumo de todas as drogas, apresentando efeito positivo apenas no uso de uma determinada droga. A Intervenção breve ${ }^{(27-30)}$ foi estratégia que se destacou neste estudo, uma vez que promove o reforço motivacional em adolescentes. Ela também é centrada no aconselhamento ao cliente realizada em um tempo restrito, buscando alterações de comportamento, tomada de decisão e comprometimento com a mudança ${ }^{(41)}$.

A escola $(74,1 \%)$ foi eleita como ambiente mais propício para o desenvolvimento das estratégias. Ainda, a efetividade de algumas das utilizadas nas escolas, como é o caso do Programa de prevenção universal do uso de álcool ${ }^{(18-19)}$ envolveram tanto os adolescentes quanto seus pais, de forma simultânea; demonstrando a importância da inclusão da família nas estratégias de prevenção ${ }^{(38-39)}$, sendo que os pais desenvolvem atitudes de restrição em relação ao consumo de drogas e o adolescente, por sua vez, apresenta maior autocontrole e redução deste uso ${ }^{(18-19,25)}$. 
O Projeto Northland ${ }^{(25)}$ além de prevenir e retardar o uso de álcool entre adolescentes, também possui potencial para reduzir as taxas de crescimento de problemas familiares. O projeto ocorreu ao longo de sete anos em três fases da vida do indivíduo: adolescência precoce, fase de transição e final da adolescência e demonstrou que a diminuição de conflitos familiares pode favorecer a presença de mecanismos de proteção do uso de álcool entre adolescentes, como o aumento da sensibilização dos pais em relação a esse uso com maior monitoramento do adolescente e reforço das consequências ${ }^{(25)}$.

O Programa Narconon ${ }^{(22)}$ é outro que tem demonstrado ser satisfatório na redução do uso de drogas no ambiente escolar; ele é composto por oito módulos: educação sobre drogas; motivação; habilidades sociais; reconhecimento; influência social; riscos; fatores de proteção contra drogas; etiologia do abuso de drogas e vícios, que objetivaram complementar as atividades de prevenção existentes em sala de aula. Também, nesse ambiente, demonstrou sucesso a Intervenção direcionada à personalidade da juventude ou ao tratamento HR (Educação Legal sobre drogas) ${ }^{(34)}$.

Ainda no ambiente escolar, outro programa eficaz que melhorou as atitudes, comportamentos e habilidades de vida dos adolescentes na intenção de não usar drogas, foi o Programa de prevenção do uso de drogas, integrado à teoria do comportamento planejado (TPB) ${ }^{(23)}$ e às habilidades de vida ${ }^{(23)}$; nele os professores utilizaram os sete critérios de qualidade de um programa de intervenção (método interativo; utilização da influência social; foco sobre normas sociais; compromisso de não utilizar drogas; intervenções comunitárias; uso de pares liderança e inclusão de habilidades de vida).

A utilização de habilidade de vida para a adolescência constitui um meio importante para prevenir ou retardar o uso de substâncias entre os adolescentes; nela, usam-se vários meios para ensinar a competência social e as habilidades de recusa ${ }^{(23,26)}$. Outros recursos que demonstraram ser eficazes para gerar mudanças de comportamento do adolescente em relação ao uso de substâncias foram a Intervenção Motivacional e o Programa de intervenção em saúde baseada na escola e utilizando a Motivação Cognitiva, Inteligência Emocional e Habilidades de Resistência ${ }^{(31,36)}$.

Para intervir nos múltiplos comportamentos de saúde um estudo ${ }^{(33)}$ descreveu efeitos positivos encontrados com aplicação de uma estratégia que integrava a realização de atividades físicas e a prevenção do uso de substâncias entre adolescentes. Essa ação fundamentou-se no Modelo Integrativo ImagemComportamento o qual afirmava que as imagens pessoais e sociais positivas servem como motivadores para o desenvolvimento da saúde.

Além dos recursos apresentados pelas estratégias de prevenção do uso de substâncias entre adolescentes até agora citadas, o uso da tecnologia como softwares interativos e a internet, é outro meio que tem refletido benefícios no que tange às ações de prevenção de álcool e/ou de outras drogas ${ }^{(29-30,32,37)}$. A eficácia desse meio para o desenvolvimento de estratégias pode estar relacionada ao menor custo e ao maior benefício, pois requer pouco treinamento, pode alcançar altos níveis de padronização e de fidelidade e é facilmente disseminado para um maior número de adolescentes ${ }^{(30)}$. Além disso, esse pode ser um meio que promove um maior interesse do adolescente, uma vez que possibilita o desenvolvimento de um 
programa animado, interativo e autônomo para a tomada decisões ${ }^{(37)}$.

Outros ambientes, além da escola, também serviram como cenário para o emprego de ações de prevenção de drogas, tais como a comunidade e o serviço de atenção à saúde $\mathrm{e}^{(27-28,31-32,37,39)}$. Também em um Centro de Apoio e Atendimento ao Adolescente (CAAA), médicos pediatras empregaram a Intervenção Breve para o Uso de Álcool e de outras drogas entre adolescentes, dividindo-os conforme o nível de consumo de substâncias no último mês (que receberam a intervenção breve) e não usuários no último mês (que receberam a orientação preventiva) ${ }^{(27)}$.

Todas as estratégias investigadas nos estudos analisados demonstraram alguma efetividade para prevenir ou para reduzir o consumo de álcool e/ou de outras drogas entre adolescentes. No entanto, sete estudos demonstraram redução do consumo de apenas uma determinada droga, como o álcool por exemplo e as estratégias abordadas por eles foram: Programa com Curriculum Unplugged ${ }^{(14-17)}$, Projeto Rumo sem abuso de drogas ${ }^{(20-21)}$ e Programa de Prevenção Universal em meio escolar se encarregar de sua vida $(\mathrm{TCYL})^{(24)}$.

Dentre as estratégias satisfatórias para prevenir ou para reduzir o consumo de álcool e/ou de outras drogas entre adolescentes, apenas o Projeto Narconon ${ }^{(22)}$ e a Intervenção Breve ${ }^{(27)}$ evidenciaram realmente ser efetivos em reduzir o uso de diferentes tipos de drogas.

Quanto ao nível de evidência, tendo em vista que constitui algo que proporciona provas de acurácia para apoiar determinações clínicas e quanto maior o nível mais forte será a evidência ${ }^{(42)}$ pode-se constatar que nesta revisão todos os estudos apresentaram níveis de evidência fortes, os quais poderão auxiliar importantes decisões clínicas no que diz respeito a prevenção ou redução do consumo de drogas entre adolescentes.

\section{CONCLUSÃO}

Ao analisar os 27 estudos que compuseram esta revisão, foram identificadas 18 estratégias de prevenção do uso de álcool e de outras drogas aplicadas para adolescentes, sendo $74,1 \%$ integralmente efetivas.

Verificou-se uma diversidade de estudos envolvendo a utilização de programas, de projetos, de intervenções, em vários países, os quais em sua maioria tinham o intuito de prevenir ou de reduzir o consumo de substâncias psicoativas em adolescentes. Em relação ao cenário de aplicação dessas estratégias, houve predominância de realização em escolas e por professores. De um modo geral, as estratégias identificadas envolviam a participação dos pais na prevenção do uso de substâncias entre seus filhos; educação sobre drogas; uso da tecnologia; emprego das habilidades de vida; aconselhamento; dentre outros; sendo valorizados como pontos importantes para a realização de tais estratégias; o envolvimento da família, da escola e o emprego da tecnologia.

Conclui-se que a utilização das estratégias de prevenção do consumo de drogas para os adolescentes é uma ação importante para minimizar essa problemática. Tais estratégias, além de prevenir ou de reduzir a 
utilização das substâncias entre os jovens podem evitar possíveis consequências decorrentes dessa prática. Dentre as estratégias investigadas nos estudos, apenas o Projeto Narconon e a Intervenção Breve demonstraram efetividade, de maneira ampla, na redução do consumo de diferentes tipos de drogas entre adolescentes, uma vez que as demais foram direcionadas à algum tipo específico de drogas ou ainda se limitaram à prevenção do consumo destas sustâncias à adolescentes não usuários.

Neste contexto, são necessários novos estudos que possam explorar a efetividade de tais estratégias voltadas à grupos de usuários de drogas ou mesmo investigações longitudinais que possam avaliar a eficácia na prevenção do consumo à longo prazo.

O presente estudo contribui para o conhecimento acerca das estratégias de prevenção ou redução do consumo de drogas para os adolescentes, no entanto, estudos de metanálise serão uteis em fortalecer os resultados explorados nas investigações analisadas.

\section{Agradecimentos}

A FAPEMIG por subsidiar o estudo (APQ 0302614) e a CAPES.

\section{REFERÊNCIAS}

1. Junqueira MAB, Nunes MJ, Miranda FJS, Castro VG, Massa DC, Bernardes ACG, et al. Prevenção ao uso abusivo de álcool no contexto escolar: relato de experiência do projeto “Recriando caminhos”. Em Extensão. 2013; 12(1): 135-43. 2. Ministério da Saúde. Secretaria Executiva. Coordenação Nacional de DST/Aids. A política do Ministério da Saúde para a atenção integral a usuários de álcool e outras drogas. Brasília (DF): Ministério da Saúde; 2003.

3. Jesús MCG, Ferriani MGC. School as a "protective fator" against drugs: perceptions of adolescents and teachers. Rev Lat-Am.Enfermagem. 2008; 16(spe):590-94.

4.Cebrid.VI Levantamento Nacional sobre o Consumo de Drogas Psicotrópicas entre Estudantes do Ensino Fundamental e Médio das Redes Pública e Privada de Ensino nas 27 Capitais Brasileiras - 2010. Carlini E.A. [et. al.] (org.) São Paulo: CEBRID- Centro Brasileiro de Informações sobre Drogas Psicotrópicas: UNIFESP - Universidade Federal de São Paulo; 2010.

5.Atanázio EA, Santos JM, Dionísio LR, Silva J, Ana AWS. Vulnerabilidade ao uso de álcool: um estudo com adolescentes das redes pública e privada de ensino. SMAD. Revista Eletrônica Saúde Mental Álcool Drogas .2013; 9(1): 11-17.

6.Brasil.Ministério da Justiça. Secretaria Nacional de Política sobre Drogas. Prevenção do Uso de Drogas: capacitação para conselheiros e lideranças comunitárias. Brasília (DF): Senad; 2013.

7.Brasil.Unidade de Pesquisas em Álcool e Drogas. Uniad. Custos Sociais decorrentes do uso indevido de drogas.s.d. Disponível em:

http://www.uniad.org.br/desenvolvimento/images/stories/publicacoes/outros/Custos\%20sociais\%20decorrentes\%20 do\%20uso\%20indevido\%20de\%20drogas.pdf.

8.Oliveira EB. Bittencourt LP, Carmo AC. A importância da família na prevenção do uso de drogas entre crianças e adolescentes: papel materno. SMAD. Revista Eletrônica Saúde Mental Álcool e Drogas. 2008; 4(2): 1-16.

9.Mariz SR, Souza LCOS, Bessa CLS, Costa FHA, Pinheiro DMQ, Brasileiro NA. Análise de estratégias em prevenção ao uso indevido de drogas entre estudantes de uma escola municipal em Campina Grande. Revista Saúde e Ciência.

2011;2(1):19-29

10.Galvão CM, Sawada NO, Trevizan MA. Revisão sistemática: recurso que proporciona a incorporação das evidências na prática de enfermagem. Rev Lat-Am.Enfermagem. 2004; 12(3): 549-56.

11. Moher D, Liberati A, Tetzlaff J, Altman DG, The PRISMA Group. Preferred Reporting Items for Systematic Reviews and Meta-Analyses: The PRISMA Statement. PLoS Med 2009; 6(6). 
12. Stetler CB, Brunell M, Giuliano KK, Morsi D, Prince L, Newell-Stokes V. Evidence-based practice and the role of nursing leadership. J Nurs Adm. 1998;28(7-8):45-53

13.Alves VS. Modelos de atenção a saúde de usuários de álcool e outras drogas: discursos políticos, saberes e práticas. Cad. Saúde Pública. 2009; 25(11):2309-29.

14.Giannotta F, Taglianti FV, Galanti MR, Scatigna M, Gaggiano F. Short-Term Mediating Factors of a School-Based Intervention to Prevent Youth Substance Use in Europe. Journal of Adolescent Health.2014;54:565-73.

15.Faggiano F , Taglianti FV, Burkhart G, Bohrn K, Cuomo L, Gregori D,et al. The effectiveness of a school-based substance abuse prevention program:18-Month follow-up of the EU-Dap cluster randomized controlled trial. Journal Elsevier.2010;108:56-64.

16.Caria MP, Faggiano F, Belloco R, Galanti MR. Effects of a School-Based Prevention Program on European Adolescents Patterns of Alcohol Use. Journal of Adolescent Health.2011; 48(2): 182-8.

17.Gabrhelik R, Duncan A, Miovsky M, Furr-Holden CD, Stastna L, Jurystova L. "Unplugged”: A school-based randomized control trial to prevent and reduce adolescent substance use in the Czech Republic. Drug and Alcohol Dependence. 2012; 124:79-87.

18.Koning IM, van den Eijnden RJ, Verdurmen JE, Engels RC, Vollebergh WA. I. A cluster randomized trial on the effects of a parent and student intervention on alcohol use in adolescents four years afterbaseline; no evidence of catchingup behavior. Journal Addictive Behaviors. 2013; 38: 2032-39.

19.Koning IM , Verdurmen JE, Engels RC, van den Eijnden RJ, Vollebergh WA. Differential Impact of a Dutch Alcohol Prevention Program Targeting Adolescents and Parents Separately and Simultaneously: Low Self-Control and Lenient Parenting at Baseline Predict Effectiveness.Prev Sci.2012; 13:278-87.

20.Sussman S, Sun P, McCuller WJ, Dent CW. Project Towards No Drug Abuse: two-year outcomes of a trial thatcompares health educator delivery to self-instruction. Preventive Medicine.2003; 37:155-62.

21.Rohrbach LA, Sun P, Sussman S. One-year follow-up evaluation of the Project Towards No Drug Abuse (TND)dissemination trial. Preventive Medicine.2010; 51:313-19.

22.Lennox RD. Cecchini MA.The NARCONON ${ }^{\mathrm{TM}}$ drug education curriculum for high school students: A nonrandomized, controlled prevention trial. Journal Substance Abuse Treatment, Prevention, and Policy.2008; 3(8):1-14. 23. Huang CM, Chien LY, Cheng CF, Guo JL. Integrating Life Skills Into a Theory-BasedDrug-Use Prevention Program: EffectivenessAmong Junior High Students in Taiwan. Journal of School Health. 2012; 82(7): 328-35.

24.Sloboda Z, Stephens RC, Stephens PC, Grey SF, Teasdale B, Hawthorne RD, et al.The Adolescent Substance Abuse Prevention Study: A randomized field trial of a universal substance abuse prevention program. Journal Elsevier. 2009; 102:1-10.

25.Perry CL, Lee S, Stigler MH, Farbakhsh K, Komro KA, Gewirtz AH, et al. The Impact of Project Northland on Selected MMPI-A Problem Behavior Scales. Journal Primary Prevent.2007;28:.449-65.

26.Eisen M, .Zellman GL, Murray DM. Evaluating the Lions-Quest "Skills for Adolescence" drugeducation programSecond-year behavior outcomes. Journal Addictive Behaviors.2003;28:883-97.

27. Micheli DD, Fisberg M, Formigoni MLOS. Estudo da efetividade da intervenção breve para o uso de álcool e outras drogas em adolescentes atendidos num serviço de assistência primária à saúde. Rev Assoc Med Bras.2004;50(3):305-

13.

28.Goti J, Diaz R, Serrano L, Gonzalez L, Calvo R, Gual A, et al. Brief intervention in substance-use among adolescent psychiatric patients: a randomized controlled trial.Eur Child Adolesc Psychiatry.2010;19:503-11.

29.Patten CA, Croghan IT, Meis TM, Decker PA, Pingree S, Colligan RC, et al. Randomized clinical trial of an Internetbased versus briefoffice intervention for adolescent smoking cessation. Patient Education and Counseling.2006; 64:249-58.

30.Doumas DM, Esp S, Turrisi R, Hausheer R, Cuffee C.A test of the efficacy of a brief,web-based personalized feedbackintervention to reduce drinking among 9th grade students. Addictive Behaviors.2014; 39:231-38.

31.Baer JS, Beadnell B, Garrett SB, Hartzler B, Wells EA, Peterson PL. Adolescent Change Language Within a Brief Motivational Intervention and Substance Use Outcomes. Journal Psychology of Addictive Behaviors. 2008;22(4):570-5. 32.Gregor MA, Shope JT, Blow FC, Maio RF, Weber JE, Nypaver MM. Feasibility of Using an Interactive Laptop Program in the Emergency Department to Prevent Alcohol Misuse Among Adolescents. Annals of emergency medicine.2003;42(2):276-84.

33.Werch CC, Moore MJ, DiClemente CC, Bledsoe R, Jobli E. A Multihealth Behavior Intervention Integrating Physical 
Activity and Substance Use Prevention for Adolescents. Prevention Science. 2005; 6(3):213-26.

34.Conrod PJ. Effectiveness of a Selective, Personality-Targeted Prevention Program for Adolescent Alcohol Useand Misuse. Jama psychiatry.2013;70(3):334-42.

35.Serrano OH, Griffin KW, Fernández JMG, Espada MOJP. Public commitment, resistance to advertising, and leisure promotion in a school-based drug abuse prevention program: a component dismantling study. J Drug Education.2013; 43(4):331-51.

36.Guo R, He Q, Shi J, Gong J, Wang H, Wang Z. Short-term Impact of Cognition-Motivation-Emotional IntelligenceResistance Skills Program on Drug Use Prevention for School Studentsin Wuhan, China. J Huazhong Univ Sci Technol.2010;30(6):720-5.

37.Walton MA, Bohnert K, Resko S, Barry KL, Chermack ST, Zucker RA et al. Computer and therapist based brief interventions among cannabis-using adolescents presenting to primary care: One year outcomes. Drug and Alcohol Dependence.2013; 132:646-53.

38.Toumbourou JW, Gregg ME, Shortt AL, Hutchinson DM, Slaviero TM. Reduction of Adolescent Alcohol Use Through FamilyeSchool Intervention: A Randomized Trial. Journal of Adolescent Health. 2013;53:778-84.

39.Milburn NG, Iribarren FJ, Rice E, Lightfoot M, Solorio R, Borus MJR, et al. A Family Intervention to Reduce Sexual Risk Behavior, Substance Use, and Delinquency Among Newly HomelessYouth. J of Adoles Health.2012; 50:358-364. 40.Primack BA, Douglas EL, Land SR, Miller E, Fine MJ. Comparison of Media Literacy and Usual Education to Prevent Tobacco Use: A Cluster-Randomized Trial. Journal of School Health.2014; 84(2):106-116.

41.Segatto ML, Pinsky I, Laranjeira R, Rezende FF, Vilela TR. Triagem e intervenção breve em pacientes alcoolizados atendidos na emergência: perspectivas e desafios. Cad. Saúde Pública.2007; 23(8):1753-1762.

42.Cruz DALM, Pimenta CAM. Prática baseada em evidências, aplicada ao raciocínio diagnóstico. Rev Latino-am Enfermagem.2005; 13(3): 415-22. 